

\title{
ESTIMÉES DES NOYAUX DE GREEN ET DE LA CHALEUR SUR LES ESPACES SYMÉTRIQUES
}

\author{
GILLES CARRON
}

\begin{abstract}
On majore les noyaux de Green et de la chaleur au dehors de la diagonale pour des opérateurs de type laplacien sur les espaces symétriques.

We provide an upper bound for the off-diagonal entries of the Green and heat kernel for Laplace-type operators on symmetric spaces.
\end{abstract}

\section{Introduction}

On considère ici un espace symétrique $X=G / K$ de type non compact. À une représentation unitaire $(\rho, V)$ de dimension finie de $K$, on associe le fibré vectoriel $G \times_{K} V$ au dessus de $X$, dont l'espace des sections lisses s'identifie à

$$
C^{\infty}(E) \simeq\left\{f \in C^{\infty}(G, V): g \in G, k \in K \Rightarrow f(g k)=\rho\left(k^{-1}\right) f(g)\right\}
$$

L'objet de cet article est un opérateur de type laplacien $G$-invariant agissant sur les sections de $E$

$$
L=\nabla^{*} \nabla+R
$$

où $\nabla$ est une connexion hermitienne $G$-invariante sur $E$ et $R$ une section $G$-invariante du fibré des endomorphismes hermitiens de $E$. Nous donnons quelques estimations de la résolvante et du noyau de la chaleur associé à $L$. Notre premier résultat est le suivant :

Théorème A. Notons $\lambda_{0}$ le bas du spectre de l'opérateur $L, o=\operatorname{Id} . K \in X$ et $G_{s}(x, y)$ le noyau de Schwartz de la résolvante $\left(L-\lambda_{0}+s^{2}\right)^{-1}$ où s est un nombre complexe tel que $s$ et $s^{2}$ aient leurs parties réelles strictement positives. Il y a une constante $C$ telle que pour tout $x \in X$ tel que $d(x, o) \geq 2$, on ait alors :

$$
\left|G_{s}(x, o)\right| \leq C e^{-\rho\left(x^{+}\right)-\operatorname{Re}(s) d(x, o)},
$$

où on a noté $x^{+}$la composante suivant $\overline{\mathfrak{a}}^{+}$de $x=g K$ dans la décomposition de Cartan $G=K e^{\bar{a}^{+}} K$ et $\rho \in \mathfrak{a}_{\mathbb{C}}^{*}$ la demi somme des racines restreintes positives associées à $\left(\mathfrak{g}_{\mathbb{C}}, \mathfrak{a}\right)$.

Le calcul explicite de $\lambda_{0}$ est en général difficile. Le bas du spectre du laplacien agissant sur les fonctions est égal à $\|\rho\|^{2}$. Concernant le laplacien de Hodge-de Rham sur les formes différentielles des calculs explicites sont faits par H. Donnelly [1981] et E. Pedon [1999; 2005] en rang 1 et par N. Lohoué et S. Medhi [2007, Appendix A] pour certains espaces hermitiens.

MSC2000: primary 53C35, 58J50; secondary 22E40.

Mots-clefs: espace symétrique, noyau de Green, noyau de la chaleur, laplacien, propagation à vitesse finie, symmetric space,

Green kernel, heat kernel, laplacian, finite-speed propagation. 
La preuve de notre estimation n'utilise que deux ingrédients, à savoir une estimation du volume de $K B(x, 1) \subset X$ et un argument désormais classique, introduit par J. Cheeger, M. Gromov et M. Taylor [Cheeger et al. 1982], de propagation à vitesse finie. Pour certains espaces localement symétriques ou à géométrie bornée, Taylor [1989] a utilisé la technique de propagation à vitesse finie pour obtenir des résultats optimaux sur les normes $L^{p} \rightarrow L^{p}$ de fonctions du laplacien.

Notre résultat est sensiblement meilleur que celui obtenu récemment par Lohoué et Mehdi [2007] à propos du laplacien de Hodge-de Rham; en utilisant un théorème de Paley-Wiener de P. Delorme [2005] et la théorie des représentations de $G$, ils obtiennent pour tout $\varepsilon \in$ ]0, 1[ l'existence d'une constante $C_{\varepsilon}$ telle que pour tout $x \in X$ tel que $d(x, o) \geq 1$,

$$
\left|G_{s}(x, o)\right| \leq C_{\varepsilon} \Phi_{0}(x) e^{-(1-\varepsilon) \operatorname{Re}(s) d(x, o)},
$$

où $\Phi_{0}$ est la fonction sphérique élémentaire de Harish-Chandra de $G$. On sait qu'il y a une constante telle que $\Phi_{0}(x) \geq C e^{-\rho\left(x^{+}\right)}$, en fait la fonction $\Phi_{0}(x) e^{\rho\left(x^{+}\right)}$croît polynomialement sur $\overline{\mathfrak{a}}^{+}$[Anker 1987].

L'approche développée par R. Mazzeo et A. Vasy utilise elle la géométrie de l'espace symétrique et une construction de parametrice reliée à cette géométrie. Il s'agit d'une méthode beaucoup plus élaborée que la nôtre mais elle fournit beaucoup plus d'informations que l'estimation ponctuelle obtenue ici. Dans le cas de l'espace symétrique $\mathrm{SL}_{3}(\mathbb{R}) / \mathrm{SO}_{3}(\mathbb{R})$, Mazzeo et Vasy [2007] ont obtenu un développement asymptotique complet de la résolvante; de plus cette méthode pourrait être généralisée à toutes les géométries asymptotiquement symétriques.

Cependant notre estimation n'est pas, en général, optimale. Par exemple pour les fonctions, on sait grâce au travail de J-P. Anker et L. Ji [1999, Theorem 4.22(i)] que pour $s>0$, l'on a une estimation de la forme

$$
C^{-1} d(x, o)^{-\beta} \Phi_{0}(x) e^{-s d(x, o)} \leq G_{s}(x, o) \leq C d(x, o)^{-\beta} \Phi_{0}(x) e^{-s d(x, o)}
$$

où si on note $\Sigma^{++}$les racines positives indivisibles et $l$ le rang de $X$ alors $\beta=\left|\Sigma^{++}\right|+(l-1) / 2$. En fait, on a l'estimation $d(x, o)^{-\beta} \Phi_{0}(x) \leq C d(x, o)^{-(l-1) / 2} e^{-\rho\left(x^{+}\right)}$. Sur les fonctions, notre estimation est donc optimale en rang 1, et en rang supérieur, elle est optimale à un facteur polynomial près; notons également que grâce à [Carron et Pedon 2004, Theorem 3.6], notre résultat est optimal pour le laplacien de Hodge-de Rham en rang 1.

Nous avons également obtenu une estimation du noyau de Schwartz de l'opérateur de la chaleur $e^{-t L}$ par la même méthode. Pour énoncer ce résultat, on rappelle quelques notations sur la structure algébrique de $X$. On note $\mathfrak{k} \subset \mathfrak{g}$ les algèbres de Lie de $K$ et $G$ et

$$
\mathfrak{g}=\mathfrak{k} \oplus \mathfrak{p}
$$

la décomposition en espaces propres de l'involution de Cartan $\theta$. Soit $\mathfrak{a} \subset \mathfrak{p}$ une sous-algèbre abélienne maximale et $\Sigma \subset \mathfrak{a}_{\mathbb{C}}^{*}$ le système restreint des racines de $(\mathfrak{g}, \mathfrak{a})$. On fixe alors $\mathfrak{a}^{+} \subset \mathfrak{a}$ une chambre de Weyl et on note $\Sigma^{+} \subset \Sigma$ le système des racines restreintes positives associées. Le rang de l'espace symétrique $X$ est $l=\operatorname{dim} \mathfrak{a}$; la dimension de l'espace symétrique $X$ est notée $d$. L'espace $\mathfrak{p}$ se décompose en

$$
\mathfrak{p}=\mathfrak{a} \oplus \bigoplus_{\alpha \in \Sigma^{+}} \mathfrak{p}_{\alpha}
$$

où si on introduit

$$
\mathfrak{n}_{\alpha}=\{n \in \mathfrak{g}: a \in \mathfrak{a} \Rightarrow \operatorname{ad}(a) n=\alpha(a)\}
$$


alors

$$
\mathfrak{p}_{\alpha}=\left\{x+\theta(x): x \in \mathfrak{n}_{\alpha}\right\} .
$$

On note aussi $m_{\alpha}=\operatorname{dim} \mathfrak{n}_{\alpha}$ et donc $\rho=\frac{1}{2} \sum_{\alpha \in \Sigma^{+}} m_{\alpha} \alpha \in \mathfrak{a}_{\mathbb{C}}^{*}$. Dans la décomposition de Cartan de $G=K e^{\overline{\mathfrak{a}}^{+}} K$, si $x=g K \in X=G / K$, on note $x^{+}$l'unique élément de $\overline{\mathfrak{a}}^{+}$tel que $g \in K e^{x^{+}} K$. Notre estimation est alors la suivante :

Théorème B. Notons $h_{t}(x, y)$ le noyau de Schwartz de l'opérateur de la chaleur $e^{-t L}$. Il existe une constante $C$ telle que pour tout $x \in X$ tel que $d(x, o) \geq 2$ on ait :

$$
\left|h_{t}(x, o)\right| \leq C e^{-\lambda_{0} t-\rho\left(x^{+}\right)-d(x, o)^{2} / 4 t} \phi_{t}(x)
$$

où

$$
\phi_{t}(x)= \begin{cases}\frac{\sqrt{t}}{d(x, o)+\sqrt{t}} & \text { si } d(x, o) \leq t, \\ \frac{d(x, o)^{(d+l) / 2-1}}{t^{(d+l-1) / 2}} \prod_{\alpha \in \Sigma^{+}}\left(\frac{1+\alpha\left(x^{+}\right)}{\frac{t}{d(x, 0)}+\alpha\left(x^{+}\right)}\right)^{m_{\alpha} / 2} & \text { si } d(x, o) \geq t .\end{cases}
$$

Cette majoration n'est également pas optimale. On peut comparer notre estimation avec celle obtenue par Lohoué et Mehdi [2007] à propos du laplacien de Hodge-de Rham ; ils obtiennent pour tout $\varepsilon \in$ ]0, 1 [ des constantes $C_{\varepsilon}$ et $A_{\varepsilon}$ telles que si $d(x, o) \geq A_{\varepsilon}$ alors

$$
\left|h_{t}(x, o)\right| \leq C_{\varepsilon} \Phi_{0}(x) e^{-\lambda_{0} t} e^{-(1-\varepsilon) d(x, o)^{2} / 4 t} t^{-\varepsilon \gamma} .
$$

Notre estimation est donc meilleure lorsque $d(x, o)$ tend vers l'infini mais bien plus mauvaise lorsque $t$ tend vers $+\infty$. Dans le cas de l'espace hyperbolique réel et du laplacien scalaire, on peut vérifier avec l'estimation de E. Davies et N. Mandouvalos [1988] que notre estimée est optimale dans le régime où $d(x, o) \geq \max \{2, t\}$.

\section{Une estimée de volume}

Proposition 2.1. Il y a des constantes strictement positives $c, C$ telles que pour tout $\varepsilon \in[0,1[$ et $x \in X$

$$
c \varepsilon^{l} e^{2 \rho\left(x^{+}\right)} \prod_{\alpha \in \Sigma^{+}}\left(\frac{\varepsilon+\alpha\left(x^{+}\right)}{1+\alpha\left(x^{+}\right)}\right)^{m_{\alpha}} \leq \operatorname{vol} K B(x, \varepsilon) \leq C \varepsilon^{l} e^{2 \rho\left(x^{+}\right)} \prod_{\alpha \in \Sigma^{+}}\left(\frac{\varepsilon+\alpha\left(x^{+}\right)}{1+\alpha\left(x^{+}\right)}\right)^{m_{\alpha}} .
$$

Démonstration. Grâce à [Anker et Ji 1999, lemme 2.1.2], nous savons que

$$
K B(x, \varepsilon) \simeq K \exp \left(B\left(x^{+}, \varepsilon\right) \cap \overline{\mathfrak{a}}^{+}\right)
$$

dans la décomposition de Cartan $X=K e^{\bar{a}^{+}}$. Ainsi si $J(h) d k d h$ est la forme volume de $X$ dans les coordonnées $(k, h) \mapsto k e^{h} K$ nous avons :

$$
\operatorname{vol} K B(x, \varepsilon)=\int_{B\left(x^{+}, \varepsilon\right) \cap \mathfrak{a}^{+}} J(h) d h .
$$


Cependant nous avons pour une constante positive $C$ :

$$
J(h)=C \prod_{\alpha \in \Sigma^{+}} \sinh ^{m_{\alpha}}(\alpha(h)) \approx C e^{2 \rho(h)} \prod_{\alpha \in \Sigma^{+}}\left(\frac{\alpha(h)}{1+\alpha(h)}\right)^{m_{\alpha}} ;
$$

c'est à dire qu'il y a une constante $\eta>0$ tel que pour tout $h$ :

$$
\eta e^{2 \rho(h)} \prod_{\alpha \in \Sigma^{+}}\left(\frac{\alpha(h)}{1+\alpha(h)}\right)^{m_{\alpha}} \leq J(h) \leq \eta^{-1} e^{2 \rho(h)} \prod_{\alpha \in \Sigma^{+}}\left(\frac{\alpha(h)}{1+\alpha(h)}\right)^{m_{\alpha}}
$$

Grâce à la preuve de [Anker et Ji 1999, lemme 2.1.6(i)], on en déduit que pour $\varepsilon \in$ ]0, 1[ et $h \in$ $B\left(x^{+}, \varepsilon\right) \cap \mathfrak{a}^{+}$, on a

$$
\rho\left(x^{+}\right)-|\rho| \leq \rho(h) \leq \rho\left(x^{+}\right)+|\rho|
$$

et pour $\alpha \in \Sigma^{+}$,

$$
\left|\alpha\left(h-x^{+}\right)\right| \leq \varepsilon / \sqrt{2}, \quad\left(1-\frac{1}{\sqrt{2}}\right)\left(1+\alpha\left(x^{+}\right)\right) \leq 1+\alpha(h) \leq 2\left(1+\alpha\left(x^{+}\right)\right), \quad \alpha(h) \leq \alpha\left(x^{+}\right)+\varepsilon .
$$

On en déduit aisément la majoration annoncée.

Pour la minoration, on considère $\Sigma^{+++}=\left\{\alpha_{1}, \ldots, \alpha_{l}\right\}$ un système de racines réduites qui est une base de $\mathfrak{a}_{\mathbb{C}}^{*}$ et $E_{1}, \ldots, E_{l}$ la base de $\mathfrak{a}$ duale à $\Sigma^{+++}$. On pose alors

$$
v=\sum_{i} E_{i}
$$

Ainsi pour $\alpha \in \Sigma^{+}$, on a $\alpha(v) \geq 1$. On a bien évidemment

$$
B\left(x^{+}+\frac{\varepsilon}{10+10|v|} v, \frac{\varepsilon}{20+20|v|}\right) \subset B\left(x^{+}, \varepsilon\right) ;
$$

or sur la boule de gauche on a pour $\alpha \in \Sigma^{+}$

$$
\alpha \geq \alpha\left(x^{+}\right)+\frac{\varepsilon}{10+10|v|} \alpha(v)-\frac{\varepsilon}{20+20|v|} \geq \alpha\left(x^{+}\right)+\frac{\varepsilon}{20+20|v|} .
$$
On obtient ainsi facilement une minoration du volume de $K B\left(x^{+}+\frac{\varepsilon}{10+10|v|} v, \frac{\varepsilon}{20+20|v|}\right)$ et donc
du volume de $K B\left(x^{+}, \varepsilon\right)$.

\section{Estimation du noyau de Green}

Ici, on étudie le noyau de Schwartz de l'opérateur $\left(L-\lambda_{0}+s^{2}\right)^{-1}$ au dehors de la diagonale où $s$ est un nombre complexe de partie réelle strictement positive. On commence par une estimée classique induite par la propriété de propagation à vitesse finie de l'opérateur $\cos \left(t \sqrt{L-\lambda_{0}}\right)$; cf. [Cheeger et al. 1982, Proposition 3.1] et aussi [Ma et Marinescu 2007, appendice D]. On considère $x \in X$ vérifiant $d(x, o) \geq 2$ et on note $A:=K B(x, 1)$.

Lemme 3.1. Soit $\sigma \in L^{2}(A, E)$ et $u:=\left(L-\lambda_{0}+s^{2}\right)^{-1} \sigma$. Alors on $a$

$$
\|u\|_{L^{2}(B(o, 1))} \leq \frac{1}{(\operatorname{Re} s)^{2}} e^{-\operatorname{Re}(s)(d(x, o)-2)}\|\sigma\|_{L^{2}} .
$$


Démonstration. En effet, on a

$$
u=\int_{0}^{\infty} \frac{e^{-s \xi}}{s} \cos \left(\xi \sqrt{L-\lambda_{0}}\right) \sigma d \xi
$$

Les hypothèses faites sur $x$ et $\sigma$ et la propriété de propagation à vitesse finie impliquent que dès que $0 \leq \xi \leq d(x, o)-2$, on a $\left\|\cos \left(\xi \sqrt{L-\lambda_{0}}\right) \sigma\right\|_{L^{2}(B(o, 1))}=0$. D'où

$$
\begin{aligned}
\|u\|_{L^{2}(B(o, 1))} & \leq \int_{d(x, o)-2}^{\infty} \frac{e^{-\operatorname{Re}(s) \xi}}{|s|}\left\|\cos \left(\xi \sqrt{L-\lambda_{0}}\right) \sigma\right\|_{L^{2}(B(o, 1))} d \xi \\
& \leq \int_{d(x, o)-2}^{\infty} \frac{e^{-\operatorname{Re}(s) \xi}}{|s|}\left\|\cos \left(\xi \sqrt{L-\lambda_{0}}\right) \sigma\right\|_{L^{2}(X)} d \xi \\
& \leq \int_{d(x, o)-2}^{\infty} \frac{e^{-\operatorname{Re}(s) \xi}}{|s|}\|\sigma\|_{L^{2}(X)} d \xi \leq \frac{1}{(\operatorname{Re} s)^{2}} e^{-\operatorname{Re}(s)(d(x, o)-2)}\|\sigma\|_{L^{2}} .
\end{aligned}
$$

On utilise alors l'estimée elliptique standard suivante :

Proposition 3.2. Soit $\lambda \in \mathbb{C}$. Il y a une constante $C$ qui dépend de $X, \lambda, L$ telle que si $r \in] 0,1]$ et si $v \in L^{2}(B(x, r), E)$ vérifie $L v=\lambda v$ alors

$$
|v(x)| \leq \frac{C}{r^{d / 2}}\|v\|_{L^{2}(B(x, r))} .
$$

On en déduit l'existence d'une constante $C$ qui ne dépend que de $X, L, s$ telle que

$$
|u(o)| \leq C e^{-\operatorname{Re}(s) d(x, o)}\|\sigma\|_{L^{2}(A)} .
$$

Cette estimation fournit une majoration de la norme de l'application linéaire

$$
T: L^{2}(A, E) \mapsto E_{o} \simeq V, \quad \sigma \rightarrow\left(L-\lambda_{0}-s^{2}\right)^{-1} \sigma(o)
$$

Et donc cela induit la même majoration de la norme de l'opérateur adjoint $T^{*}: V \simeq E_{o} \rightarrow L^{2}(A, E)$ qui est défini pour $v \in E_{o}$ par :

$$
\left(T^{*} v\right)(y)=G_{\bar{s}}(y, o) v
$$

On obtient donc :

$$
\int_{K B(x, 1)}\left|G_{\bar{S}}(y, o)\right|^{2} d y \leq C e^{-2 \operatorname{Re}(s) d(x, o)} .
$$

Lemme 3.3. il y a une constante $C$ qui ne dépend que de $X$ telle que si $f \in L^{1}(A)$ alors il existe $k \in K$ tel que

$$
\|f\|_{L^{1}\left(B\left(k x, \frac{1}{4}\right)\right)} \leq C e^{-2 \rho\left(x^{+}\right)}\|f\|_{L^{1}(A)} .
$$

Démonstration. En effet,

$$
\begin{aligned}
\int_{K B\left(x, \frac{1}{4}\right)}\left(\int_{B\left(y, \frac{1}{2}\right)}|f|(z) d z\right) d y & =\int_{K B(x, 1)} \operatorname{vol}\left(B\left(z, \frac{1}{2}\right) \cap K B\left(x, \frac{1}{4}\right)\right)|f|(z) d z \\
& \leq \operatorname{vol} B\left(0, \frac{1}{2}\right) \int_{K B(x, 1)}|f|(z) d z
\end{aligned}
$$


On en déduit donc l'existence d'un $y \in K B\left(x, \frac{1}{4}\right)$ tel que

$$
\operatorname{vol} K B\left(x, \frac{1}{4}\right)\|f\|_{L^{1}(B(y, 1 / 2))} \leq C\|f\|_{L^{1}} .
$$

Il y a donc un $k \in K$ tel que $d(y, k x) \leq \frac{1}{4}$, d'où $B\left(k x, \frac{1}{4}\right) \subset B\left(y, \frac{1}{2}\right)$. Maintenant, grâce à l'estimée donnée par la proposition 2.1, $\operatorname{soit} \operatorname{vol}\left(K B\left(x, \frac{1}{4}\right)\right) \approx e^{2 \rho\left(x^{+}\right)}$, on obtient bien le résultat annoncé.

On en déduit donc l'existence d'un $k \in K$ tel que

$$
\int_{B(k x, 1 / 4)}\left|G_{\bar{s}}(y, o)\right|^{2} d y \leq C e^{-2 \operatorname{Re}(s) d(x, o)-2 \rho\left(x^{+}\right)} .
$$

Les mêmes estimées elliptiques entrânent alors

$$
\left|G_{\bar{s}}(k x, o)\right|=\left|G_{s}(o, x)\right| \leq C e^{-\operatorname{Re}(s) d(x, o)-\rho\left(x^{+}\right)},
$$

ce qui démontre le théorème $\mathrm{A}$.

\section{Estimation du noyau de la chaleur}

On étudie maintenant de la même façon le noyau de Schwartz de l'opérateur de la chaleur $e^{-t L}$ au dehors de la diagonale. On considère donc $x \in X$ et $\varepsilon>0$ tels que $d(x, o) \geq 2 \varepsilon$. On note $A:=K B(x, \varepsilon)$. Nous commençons par le même type d'estimations :

Lemme 4.1. Soit $\sigma \in L^{2}(A, E)$ et $f_{t}:=e^{-t L} \sigma$. Alors on a

$$
\left\|f_{t}\right\|_{L^{2}(B(o, \varepsilon))} \leq \frac{e^{-\lambda_{0} t}}{\sqrt{\pi t}} \int_{d(x, o)-2 \varepsilon}^{\infty} e^{-\xi^{2} / 4 t} d \xi\|\sigma\|_{L^{2}} .
$$

Cette estimation se montre de la même façon que l'estimée du lemme 3.1, en partant de la formule :

$$
f_{t}=\frac{e^{-\lambda_{0} t}}{\sqrt{\pi t}} \int_{0}^{\infty} e^{-\xi^{2} / 4 t} \cos \left(\xi \sqrt{L-\lambda_{0}}\right) \sigma d \xi
$$

Maintenant, on utilise l'estimation parabolique suivante :

Proposition 4.2. Il y a une constante $C$ (qui ne dépend que de $X, L$ ) telle que si $r \in] 0,1]$ et si $v \in$ $L^{2}\left(\left[t-r^{2}, t\right] \times B(x, r), E\right)$ est une solution de l'équation:

$$
\frac{\partial}{\partial t} v+L v=0
$$

alors

$$
|v(t, x)|^{2} \leq \frac{C}{r^{d+2}} \int_{t-r^{2}}^{t}\left(\int_{B(x, r)}|v(\tau, y)|^{2} d y\right) d \tau .
$$

Ceci provient du fait que si on note $\mu$ la plus petite valeur propre de l'opérateur $R$ dans la formule (1-1) alors la fonction $u$ définie par

$$
u(t, x)=|v(t, x)| e^{\mu t}
$$

vérifie

$$
\frac{\partial}{\partial t} u+\Delta u \leq 0
$$


Les inégalités paraboliques de J. Moser impliquent alors ce résultat; voir [Moser 1964], [Grigor'yan 1994, Theorem 3.1] ou [Saloff-Coste 1992, Theorem 5.1].

On en déduit l'existence d'une constante $C$ telle que

$$
\left|f_{t}(o)\right|^{2} \leq \frac{C}{\varepsilon^{d+2}} \int_{\left[t-\varepsilon^{2}, t\right] \times B(o, \varepsilon)}\left|f_{\tau}(y)\right|^{2} d \tau d y .
$$

Avec l'estimation

$$
\int_{A}^{\infty} e^{-\xi^{2} / 4 t} d \xi=\sqrt{t} \int_{A^{2} / 4 t}^{\infty} e^{-v} \frac{d v}{\sqrt{v}} \leq \frac{C \sqrt{t}}{A / \sqrt{t}+1} e^{-A^{2} / 4 t},
$$

on obtient, pour $\varepsilon \in] 0,1]$ et $t \geq 2 \varepsilon^{2}$ et $d(x, o) \geq 2$, l'estimée suivante :

$$
\left|f_{t}(o)\right| \leq C \varepsilon^{-d / 2} \frac{\sqrt{t}}{d(x, o)+\sqrt{t}} e^{-\lambda_{0} t-(d(x, o)-2 \varepsilon)^{2} / 4 t}\|\sigma\|_{L^{2}} .
$$

Avec les mêmes arguments que ceux utilisés dans la preuve du théorème $\mathrm{A}$, on en déduit :

$$
\left(\int_{K B(x, \varepsilon)}\left|h_{t}(y, o)\right|^{2} d y\right)^{1 / 2} \leq C \varepsilon^{-d / 2} \frac{\sqrt{t}}{d(x, o)+\sqrt{t}} e^{-\lambda_{0} t-(d(x, o)-2 \varepsilon)^{2} / 4 t} .
$$

La même argumentation basée sur le lemme 3.3 permet de trouver $k \in K$ tel que

$$
\left(\int_{B(k x, \varepsilon / 4)}\left|h_{t}(y, o)\right|^{2} d y\right)^{1 / 2} \leq C(\operatorname{vol} K B(x, \varepsilon))^{-1 / 2} \frac{\sqrt{t}}{d(x, o)+\sqrt{t}} e^{-\lambda_{0} t-(d(x, o)-2 \varepsilon)^{2} / 4 t} .
$$

Les mêmes estimées paraboliques donnent alors la majoration suivante : pour $\varepsilon \in] 0,1 / 2], t \geq 3 \varepsilon^{2}$ et $d(x, o) \geq 2$, on a

$$
\left|h_{t}(k x, o)\right|=\left|h_{t}(x, o)\right| \leq C\left(\varepsilon^{d} \operatorname{vol} K B(x, \varepsilon)\right)^{-1 / 2} \frac{\sqrt{t}}{d(x, o)+\sqrt{t}} e^{-\lambda_{0} t-(d(x, o)-2 \varepsilon)^{2} / 4 t} .
$$

Or nous avons

$$
\frac{(d(x, o)-2 \varepsilon)^{2}}{4 t}=\frac{d(x, o)^{2}}{4 t}-\frac{d(x, o) \varepsilon}{t}+\frac{\varepsilon^{2}}{t} .
$$

Donc lorsque $d(x, o) \leq t$ on choisit $\varepsilon=\frac{1}{100}$ et on obtient la majoration :

$$
\left|h_{t}(x, o)\right| \leq C \frac{\sqrt{t}}{d(x, o)+\sqrt{t}} e^{-\lambda_{0} t-d(x, o)^{2} / 4 t-\rho\left(x^{+}\right)}
$$

Lorsque $d(x, o) \geq t$ on choisit $\varepsilon=\frac{t}{100 d(x, o)}$ et on obtient pour $d(x, o) \geq 2$

$$
\begin{aligned}
\left|h_{t}(x, o)\right| & \leq \frac{C \sqrt{t}}{d(x, o)}\left(\frac{d(x, o)}{t}\right)^{(d+l) / 2} \prod_{\alpha \in \Sigma^{+}}\left(\frac{1+\alpha\left(x^{+}\right)}{\frac{t}{100 d(x, o)}+\alpha\left(x^{+}\right)}\right)^{m_{\alpha} / 2} e^{-\lambda_{0} t-d(x, o)^{2} / 4 t-\rho\left(x^{+}\right)} \\
& \leq \frac{C d(x, o)^{(d+l) / 2-1}}{t^{(d+l-1) / 2}} \prod_{\alpha \in \Sigma^{+}}\left(\frac{1+\alpha\left(x^{+}\right)}{\frac{t}{d(x, o)}+\alpha\left(x^{+}\right)}\right)^{m_{\alpha} / 2} e^{-\lambda_{0} t-d(x, o)^{2} / 4 t-\rho\left(x^{+}\right),}
\end{aligned}
$$

ce qui termine la démonstration du théorème $\mathrm{B}$. 


\section{Applications}

Dans [Carron et Pedon 2004], une estimation du prolongement analytique de la résolvante avait été obtenue ; cependant les méthodes rudimentaires utilisées ici ne permettent pas d'obtenir un tel résultat. Néanmoins, nos estimées, comme celles de Lohoué et Mehdi, permettent une estimation inférieure du bas du spectre de l'opérateur $L$ sur des espaces localement symétriques $\Gamma \backslash G / K$ où $\Gamma \subset G$ est un sous-groupe discret sans torsion.

Définition. (cf. [Carron et Pedon 2004, Theorem 2.7]) Soit $\Gamma \subset G$ est un sous-groupe discret sans torsion. On note $\tilde{\delta}(\Gamma)$ l'exposant critique modifié de $\Gamma$ qui est l'exposant critique de la série de Poincaré :

c'est à dire :

$$
\sum_{\gamma \in \Gamma} e^{-\rho\left(\gamma^{+}\right)-s d(\gamma(o), o)}
$$

$$
\tilde{\delta}(\Gamma)=\inf \left\{s \in \mathbb{R}: \sum_{\gamma \in \Gamma} e^{-\rho\left(\gamma^{+}\right)-s d(\gamma(o), o)}<\infty\right\} .
$$

Notons $G_{s}^{0}(x, y)$ le noyau de Green de l'opérateur $\left(\Delta-|\rho|^{2}+s^{2}\right)^{-1}$ agissant sur les fonctions. Grâce à notre estimation et à l'estimation inférieure de $G^{0}$ dans [Anker et Ji 1999, Theorem 4.2.2], on sait que pour tout $s>0$ et $\eta \in] 0, s\left[\right.$, il y a une constante $C_{s, \eta}$ tel que pour tout $x, y \in X$,

$$
\left|G_{s}(x, y)\right| \leq C_{s, \eta} G_{s-\eta}^{0}(x, y) .
$$

Le même raisonnement que celui utilisé pour démontrer [Carron et Pedon 2004, Theorem 2.7] montre que :

Théorème 5.1. Notons toujours $\lambda_{0}$ le bas du spectre de l'opérateur $L$ sur $X=G / K$.

(i) Si $\tilde{\delta}(\Gamma)>0$ alors le bas du spectre de $L$ sur $\Gamma \backslash G / K$ est minoré par $\lambda_{0}-(\tilde{\delta}(\Gamma))^{2}$.

(ii) Si $\tilde{\delta}(\Gamma) \leq 0$ alors le bas du spectre de $L$ sur $\Gamma \backslash G / K$ est minoré par $\lambda_{0}$. (iii) Si $\tilde{\delta}(\Gamma) \leq 0$ et si le rayon d'injectivité de $\Gamma \backslash G / K$ est non-majoré, i.e., $\sup _{x \in X} \inf _{\gamma \in \Gamma} d(x, \gamma(x))=\infty$,
alors le bas du spectre de L est $\lambda_{0}$.

Remarques 5.2. (i) Cet exposant critique modifié se compare aisément à l'exposant critique de $\Gamma$, à savoir à $\delta(\Gamma)$, l'exposant critique de la série

$$
\sum_{\gamma \in \Gamma} e^{-s d(\gamma(o), o)}
$$

Si on note $\rho_{\min }=\inf _{H \in \mathfrak{a}_{+}} \rho(h) /|h|$ alors

$$
\rho_{\min }+\tilde{\delta}(\Gamma) \leq \delta(\Gamma) \leq|\rho|+\tilde{\delta}(\Gamma)
$$

ce qui permet de ré-obtenir le résultat dans [Lohoué et Mehdi 2007, Theorem 6.1].

(ii) Lorsque $\tilde{\delta}(\Gamma)<\sqrt{\lambda_{0}}$, ce résultat implique que le noyau $L^{2}$ de $L$ sur $\Gamma \backslash G / K$ est trivial. Il est cependant difficile d'obtenir des calculs explicites de $\lambda_{0}$. Concernant le laplacien de Hodge-de Rham sur les formes différentielles des calculs explicites se trouvent dans [Donnelly 1981; 1999; 2005] en rang 1 et dans [Lohoué et Mehdi 2007, Appendix A] pour certains espaces hermitiens. 


\section{Remerciements}

Je remercie ici E. Pedon et M. Olbrich pour leurs commentaires très utiles. Je remercie également le rapporteur pour ses remarques perspicaces. Je bénéficie du support partiel du projet ANR GeomEinstein 06-BLAN-0154.

\section{References}

[Anker 1987] J.-P. Anker, "La forme exacte de l'estimation fondamentale de Harish-Chandra", C. R. Acad. Sci. Paris Sér. I Math. 305:9 (1987), 371-374. MR 89i:22016 Zbl 0636.22005

[Anker et Ji 1999] J.-P. Anker et L. Ji, "Heat kernel and Green function estimates on noncompact symmetric spaces", Geom. Funct. Anal. 9:6 (1999), 1035-1091. MR 2001b:58038 Zbl 0942.43005

[Carron et Pedon 2004] G. Carron et E. Pedon, "On the differential form spectrum of hyperbolic manifolds", Ann. Sc. Norm. Super. Pisa Cl. Sci. (5) 3:4 (2004), 705-747. MR 2005j:58041 Zbl 1170.53309

[Cheeger et al. 1982] J. Cheeger, M. Gromov et M. Taylor, "Finite propagation speed, kernel estimates for functions of the Laplace operator, and the geometry of complete Riemannian manifolds", J. Differential Geom. 17:1 (1982), 15-53. MR 84b:58109 Zbl 0493.53035

[Davies et Mandouvalos 1988] E. B. Davies et N. Mandouvalos, "Heat kernel bounds on hyperbolic space and Kleinian groups”, Proc. London Math. Soc. (3) 57:1 (1988), 182-208. MR 89i:58137 Zbl 0643.30035

[Delorme 2005] P. Delorme, "Sur le théorème de Paley-Wiener d'Arthur", Ann. of Math. (2) 162:2 (2005), 987-1029. MR 2006g:22009 Zbl 1121.22002

[Donnelly 1981] H. Donnelly, "The differential form spectrum of hyperbolic space”, Manuscripta Math. 33:3-4 (1981), 365385. MR 82f:58085 Zbl 0464.58020

[Grigor'yan 1994] A. Grigor'yan, "Heat kernel upper bounds on a complete non-compact manifold", Rev. Mat. Iberoamericana 10:2 (1994), 395-452. MR 96b:58107

[Lohoué et Mehdi 2007] N. Lohoué et S. Mehdi, "Estimées du noyau de la chaleur pour les formes différentielles sur les espaces symétriques et $L^{2}$-cohomologie des espaces localement symétriques", C. R. Math. Acad. Sci. Paris 345:3 (2007), 119-122. MR 2009f:58037 Zbl 05182217

[Ma et Marinescu 2007] X. Ma et G. Marinescu, Holomorphic Morse inequalities and Bergman kernels, Progress in Mathematics 254, Birkhäuser, Basel, 2007. MR 2008g:32030 Zbl 1135.32001

[Mazzeo et Vasy 2007] R. Mazzeo et A. Vasy, "Scattering theory on SL(3)/SO(3): connections with quantum 3-body scattering”, Proc. Lond. Math. Soc. (3) 94:3 (2007), 545-593. MR 2008g:43018 Zbl 1117.43009

[Moser 1964] J. Moser, “A Harnack inequality for parabolic differential equations”, Comm. Pure Appl. Math. 17 (1964), 101134. MR 28 \#2357 Zbl 0149.06902

[Pedon 1999] E. Pedon, "Harmonic analysis for differential forms on complex hyperbolic spaces", J. Geom. Phys. 32:2 (1999), 102-130. MR 2000j:22013 Zbl 0935.22010

[Pedon 2005] E. Pedon, "The differential form spectrum of quaternionic hyperbolic spaces", Bull. Sci. Math. 129:3 (2005), 227-265. MR 2005m:58065 Zbl 1070.22005

[Saloff-Coste 1992] L. Saloff-Coste, "Uniformly elliptic operators on Riemannian manifolds", J. Differential Geom. 36:2 (1992), 417-450. MR 93m:58122 Zbl 0735.58032

[Taylor 1989] M. E. Taylor, " $L^{p}$-estimates on functions of the Laplace operator", Duke Math. J. 58:3 (1989), 773-793. MR 91d:58253 Zbl 0691.58043

Received 15 Sep 2009. Revised 25 Jan 2010. Accepted 22 Feb 2010.

GILlES CARRON: Gilles.Carron@univ-nantes.fr

Laboratoire de Mathématiques Jean Leray (UMR 6629), Université de Nantes, 2, rue de la Houssinière, B.P. 92208 , 44322 Nantes Cedex 3, France

http://www.math.sciences.univ-nantes.fr/ carron/ 


\title{
Analysis \& PDE
}

pjm.math.berkeley.edu/apde

EDITORS

\author{
EDITOR-IN-CHIEF \\ Maciej Zworski \\ University of California \\ Berkeley, USA
}

BOARD OF EDITORS

\begin{tabular}{|c|c|c|c|}
\hline Michael Aizenman & $\begin{array}{l}\text { Princeton University, USA } \\
\text { aizenman@math.princeton.edu }\end{array}$ & Nicolas Burq & $\begin{array}{l}\text { Université Paris-Sud 11, France } \\
\text { nicolas.burq@math.u-psud.fr }\end{array}$ \\
\hline Luis A. Caffarelli & $\begin{array}{l}\text { University of Texas, USA } \\
\text { caffarel@math.utexas.edu }\end{array}$ & un-Yung Alice Chang & $\begin{array}{l}\text { Princeton University, USA } \\
\text { chang@math.princeton.edu }\end{array}$ \\
\hline Michael Christ & $\begin{array}{l}\text { University of California, Berkeley, USA } \\
\text { mchrist@math.berkeley.edu }\end{array}$ & Charles Fefferman & $\begin{array}{l}\text { Princeton University, USA } \\
\text { cf@math.princeton.edu }\end{array}$ \\
\hline Ursula Hamenstaedt & $\begin{array}{l}\text { Universität Bonn, Germany } \\
\text { ursula@math.uni-bonn.de }\end{array}$ & Nigel Higson & $\begin{array}{l}\text { Pennsylvania State Univesity, USA } \\
\text { higson@math.psu.edu }\end{array}$ \\
\hline Vaughan Jones & $\begin{array}{l}\text { University of California, Berkeley, USA } \\
\text { vfr@math.berkeley.edu }\end{array}$ & Herbert Koch & $\begin{array}{l}\text { Universität Bonn, Germany } \\
\text { koch@math.uni-bonn.de }\end{array}$ \\
\hline Izabella Laba & $\begin{array}{l}\text { University of British Columbia, Canada } \\
\text { ilaba@math.ubc.ca }\end{array}$ & Gilles Lebeau & $\begin{array}{l}\text { Université de Nice Sophia Antipolis, France } \\
\text { lebeau@unice.fr }\end{array}$ \\
\hline László Lempert & $\begin{array}{l}\text { Purdue University, USA } \\
\text { lempert@math.purdue.edu }\end{array}$ & Richard B. Melrose & $\begin{array}{l}\text { Massachussets Institute of Technology, USA } \\
\text { rbm@math.mit.edu }\end{array}$ \\
\hline Frank Merle & $\begin{array}{l}\text { Université de Cergy-Pontoise, France } \\
\text { Frank.Merle@u-cergy.fr }\end{array}$ & William Minicozzi II & $\begin{array}{l}\text { Johns Hopkins University, USA } \\
\text { minicozz@math.jhu.edu }\end{array}$ \\
\hline Werner Müller & $\begin{array}{l}\text { Universität Bonn, Germany } \\
\text { mueller@math.uni-bonn.de }\end{array}$ & Yuval Peres & $\begin{array}{l}\text { University of California, Berkeley, USA } \\
\text { peres@stat.berkeley.edu }\end{array}$ \\
\hline Gilles Pisier & $\begin{array}{l}\text { Texas A\&M University, and Paris } 6 \\
\text { pisier@math.tamu.edu }\end{array}$ & Tristan Rivière & $\begin{array}{l}\text { ETH, Switzerland } \\
\text { riviere@math.ethz.ch }\end{array}$ \\
\hline Igor Rodnianski & $\begin{array}{l}\text { Princeton University, USA } \\
\text { irod@ math.princeton.edu }\end{array}$ & Wilhelm Schlag & $\begin{array}{l}\text { University of Chicago, USA } \\
\text { schlag@ math.uchicago.edu }\end{array}$ \\
\hline Sylvia Serfaty & $\begin{array}{l}\text { New York University, USA } \\
\text { serfaty@ cims.nyu.edu }\end{array}$ & Yum-Tong Siu & $\begin{array}{l}\text { Harvard University, USA } \\
\text { siu@math.harvard.edu }\end{array}$ \\
\hline Terence Tao & $\begin{array}{l}\text { University of California, Los Angeles, USA } \\
\text { tao@math.ucla.edu }\end{array}$ & Michael E. Taylor & $\begin{array}{l}\text { Univ. of North Carolina, Chapel Hill, USA } \\
\text { met@ math.unc.edu }\end{array}$ \\
\hline Gunther Uhlmann & $\begin{array}{l}\text { University of Washington, USA } \\
\text { gunther@math.washington.edu }\end{array}$ & András Vasy & $\begin{array}{l}\text { Stanford University, USA } \\
\text { andras@math.stanford.edu }\end{array}$ \\
\hline an Virgil Voiculescu & $\begin{array}{l}\text { University of California, Berkeley, USA } \\
\text { dvv@ math.berkeley.edu }\end{array}$ & Steven Zelditch & $\begin{array}{l}\text { Johns Hopkins University, USA } \\
\text { szelditch@math.jhu.edu }\end{array}$ \\
\hline
\end{tabular}

\section{PRODUCTION}

apde@mathscipub.org

Paulo Ney de Souza, Production Manager Sheila Newbery, Production Edito

Silvio Levy, Senior Production Editor

See inside back cover or pjm.math.berkeley.edu/apde for submission instructions.

The subscription price for 2010 is US \$120/year for the electronic version, and \$180/year for print and electronic. Subscriptions, requests for back issues from the last three years and changes of subscribers address should be sent to Mathematical Sciences Publishers, Department of Mathematics, University of California, Berkeley, CA 94720-3840, USA.

Analysis \& PDE, at Mathematical Sciences Publishers, Department of Mathematics, University of California, Berkeley, CA 94720-3840 is published continuously online. Periodical rate postage paid at Berkeley, CA 94704, and additional mailing offices.

APDE peer-review and production is managed by EditFLOW ${ }^{\mathrm{TM}}$ from Mathematical Sciences Publishers.

PUBLISHED BY

mathematical sciences publishers

http://www.mathscipub.org

A NON-PROFIT CORPORATION

Typeset in IATEX $_{\mathrm{E}}$

Copyright @2010 by Mathematical Sciences Publishers 


\section{ANALYSIS \& PDE}

\section{Volume $3 \quad$ No. $2 \quad 2010$}

Polynomials with no zeros on the bidisk

\section{GREG KNESE}

Local wellposedness for the 2+1-dimensional monopole equation Magdalena CZubaK

Regularity of almost periodic modulo scaling solutions for mass-critical NLS and applications DONG LI and XIAOYI ZHANG

Estimées des noyaux de Green et de la chaleur sur les espaces symétriques GILLES CARRON

Lower bounds for resonances of infinite-area Riemann surfaces 